\title{
MODULI SPACES OF SURFACES
}

\author{
YI HUANG
}

(Received 26 January 2015; first published online 10 April 2015)

\begin{abstract}
2010 Mathematics subject classification: primary 32G15; secondary 58D27, 30F60.
Keywords and phrases: Riemann surfaces, hyperbolic surfaces, Teichmüller theory, moduli space theory, Weil-Petersson geometry, McShane identity.
\end{abstract}

Moduli space theory and Teichmüller space theory are concerned with Riemann surfaces. The moduli space $\mathcal{M}(R)$ of a topological surface $R$ (with negative Euler characteristic) is the space of all possible Riemann surface structures on $R$ up to homeomorphism. Similarly, the Teichmüller space $\mathcal{T}(R)$ is the space of all possible Riemann surface structures up to homotopy. The Teichmüller space is the universal cover of the moduli space.

One key idea in the hyperbolic surface approach to Teichmüller theory is to decompose hyperbolic surfaces into fundamental pieces, such as pairs of pants or ideal triangles, and to parametrise the ways in which these pieces may be reconstituted to form a hyperbolic surface. Decomposing into pairs of pants leads to the development of Fenchel-Nielsen coordinates [1] for $\mathcal{T}(R)$, and decomposing into ideal triangles leads to Penner's $\lambda$-length coordinates [8] and Thurston's shearing length coordinates [2].

Riemann surfaces with punctures on their (smooth) borders uniformise to crowned hyperbolic surfaces, which are complete finite-area hyperbolic surfaces with geodesic borders. We generalise Fenchel-Nielsen, Penner's $\lambda$-length and Thurston's shearing coordinate systems for Teichmüller spaces of crowned hyperbolic surfaces by decomposing these surfaces into pairs of pants and ideal triangles. This often results in mixed coordinates which are part Fenchel-Nielsen, part Penner's $\lambda$-lengths.

In [6], Mirzakhani establishes a type of trigonometric identity, called a McShane identity [5], for genus- $g$ hyperbolic surfaces with $n$ labelled geodesic borders of fixed lengths $L_{1}, \ldots, L_{n}$. She utilises these identities to derive a recursion in the Weil-Petersson volume $\operatorname{Vol}_{g, n}\left(L_{1}, \ldots, L_{n}\right)$ of the moduli space $\mathcal{M}_{g, n}\left(L_{1}, \ldots, L_{n}\right)$ of such surfaces and the volumes of moduli spaces of surfaces with lower genus and/or

Thesis submitted to the University of Melbourne in June 2014; degree approved on 15 September 2014; supervisor: Paul Norbury.

(c) 2015 Australian Mathematical Publishing Association Inc. 0004-9727/2015 \$16.00 


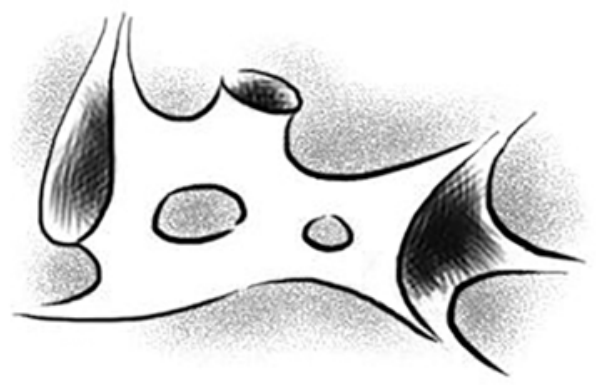

FIGURE 1. A crowned hyperbolic surface.

fewer boundary components. We establish McShane identities for crowned hyperbolic surfaces (see Figure 1), and generalise the definition of Weil-Petersson forms for moduli spaces of crowned hyperbolic spaces, computing explicit presentations for these forms in terms of our mixed coordinates. We combine these results to express a key step in Mirzakhani's volume recursion derivation in terms of integrals over moduli spaces of crowned surfaces.

The final chapter of the thesis summarises our main results in [3] and [4]. The former paper derives McShane identities for closed surfaces with one marked point. In the latter, we describe an algorithm for generating the simple length spectrum of any three-cusped projective plane and use this algorithm to:

(i) show that the maximum of the systole (shortest-geodesic) function on the moduli space of three-cusped projective planes is $2 \operatorname{arcsinh}(2)$;

(ii) prove the surprising result that the asymptotic growth rate of the one-sided simple geodesics on three-cusped projective planes is not polynomial-in contrast to the polynomial growth rate for orientable surfaces [7, 9];

(iii) derive a McShane identity for three-cusped projective planes.

\section{References}

[1] W. Abikoff, The Real Analytic Theory of Teichmüller Space, Lecture Notes in Mathematics, 820 (Springer, Berlin, 1980).

[2] A. Fathi, F. Laudenbach and V. Poénaru, Thurston's work on surfaces, Mathematical Notes, 48 (Princeton University Press, Princeton, NJ, 2012), translated from the 1979 French original by Djun M. Kim and Dan Margalit.

[3] Y. Huang, 'A McShane-type identity for closed surfaces'. Nagoya Math. J. to appear, arXiv:1203.3860.

[4] Y Huang and P. Norbury, Simple geodesics and markoff quads. arXiv:1312.7089.

[5] G. McShane, 'Simple geodesics and a series constant over Teichmuller space', Invent. Math. 132(3) (1998), 607-632.

[6] M. Mirzakhani, 'Simple geodesics and Weil-Petersson volumes of moduli spaces of bordered Riemann surfaces', Invent. Math. 167(1) (2007), 179-222.

[7] M. Mirzakhani, 'Growth of the number of simple closed geodesics on hyperbolic surfaces', Ann. of Math. (2) 168(1) (2008), 97-125. 
[8] R. C. Penner, Decorated Teichmüller Theory, QGM Master Class Series (European Mathematical Society (EMS), Zürich, 2012), with a foreword by Yuri I. Manin.

[9] I. Rivin, 'Simple curves on surfaces', Geom. Dedicata 87(1-3) (2001), 345-360.

YI HUANG, University of Melbourne, Victoria 3010, Australia

e-mail: huay@ms.unimelb.edu.au 